\title{
A propósito de la inserción laboral. Implicaciones de las nuevas políticas de empleo para el Trabajo Social
}

\author{
Regarding labour market integration. Implications of new employment \\ policies for Social Work
}

\author{
Judit FonT REDOLAD \\ Universidad de Girona (España) \\ judit.font@udg.edu \\ José Antonio LANGARITA AdIEGO \\ Universidad de Girona (España) \\ josan.langarita@udg.edu
}

Recibido: $14 / 05 / 2014$

Revisado: 04/07/2014

Aceptado: 16/02/2015

Disponible on line: $10 / 06 / 2015$

\section{Resumen}

Este artículo contribuye al debate sobre las implicaciones para el Trabajo Social de los nuevos discursos y estrategias de intervención que rodean las políticas de empleo en el Estado español. Así mismo, presenta un análisis de los conflictos, derivados de las orientaciones ideológicas que acompañan los protocolos de intervención de los profesionales. En esta línea, tiene por objeto revisar la construcción del discurso que inunda las políticas de ocupación desde la mirada del Trabajo Social, y ahondar en las repercusiones de estos discursos en la intervención profesional. Para ello se propone un análisis cualitativo derivado del estudio documental, la observación directa y la relación con profesionales y usuarios del ámbito de la orientación para el empleo. Finalmente, presenta algunas propuestas que pretenden contribuir a la mejora de la atención de los usuarios de los servicios de orientación y conducir el estudio del desempleo al análisis de la estructura social y no tanto a la problematización de las realidades individuales.

Palabras clave: inserción laboral, desempleo, exclusión social, estado de bienestar, políticas de empleo.

\begin{abstract}
This article contributes to the debate on the implications for Social Work of new discourses and intervention strategies regarding employment policies in Spain. It also presents an analysis of conflicts arising from ideological orientations, which accompanies protocols of professional intervention. Furthermore, it aims to revise the construction of discourse that floods occupation policies from the perspective of social work, and deepen the impact of these discourses in professional intervention. This research implements a qualitative approach through a desk study, direct observation. Finally, some proposals are presented to help improving the answer to the users of counseling and unemployment services as well as focusing unemployment studies on to analyze the social structure rather than emphasize individual problems.
\end{abstract}

Keywords: labor integration, unemployment, social exclusion, welfare state, employment policies.

Referencia normalizada: Font Redolad, J., y Langarita Adiego, J. A. (2015): «A propósito de la inserción laboral. Implicaciones de las nuevas políticas de empleo para el Trabajo Social». Cuadernos de Trabajo Social, 28(1): 61-68.

Sumario: Introducción. 1. Método. 2. Resultados y discusión. 3. Conclusiones. 4. Referencias bibliográficas.

\section{Introducción}

En las sociedades occidentales postfordistas se produce una nueva configuración de la relación entre Estado y mercado como resultado de la intensificación de las políticas neoliberales. Estas transformaciones conducen a nuevos procesos de desmantelamiento del Estado del bienestar, a la de flexibilización y desregulación del mercado de trabajo, a la reformulación y recorte de los sistemas de protección y de seguridad social, y al debilitamiento de los mecanismos tradicionales de organización y movilización social. La in- 
fluencia constante e intensiva de estos elementos ha producido un incremento de la desigualdad social que amplia los contornos de marginación y exclusión social.

El crecimiento del paro, cada vez más crónico y de perfil estructural, deja entrever un nuevo contexto de crisis de la sociedad salarial, tal y como auguraba Robert Castel (1997). Se dibuja así, un nuevo escenario con un gran número de población fuera del mercado laboral y sin el aporte salarial como elemento básico de subsistencia económica y posicionamiento social. La ubicación en relación al mercado laboral es ahora el principal factor generador de vulnerabilidad, no sólo con respecto al binomio paro-ocupación, sino también por la misma participación precaria e insegura en el mercado de trabajo de una buena parte de la población. Esta realidad pone en cuestión la inserción laboral como instrumento histórico de inclusión social.

El pacto social, de inspiración keynesiana resultante del fordismo, posicionó el trabajo como el aspecto central para la subsistencia económica y social y como condición de acceso a otros derechos sociales tales como: pensiones, prestaciones por desempleo, acceso a la sanidad, etc. El trabajo asalariado era el punto de partida para la participación social en un sentido amplio, es decir, para posicionarse en la estructura de clases, de generación de sentimiento de pertenencia, de producción de identidad y de reconocimiento social (Castel, 1997; Sennet, 2000).

En este marco económico, social y político, el Estado se erigió como el ente encargado de desarrollar unas políticas de empleo que, por un lado, aseguraran la adecuación entre la oferta y la demanda de trabajo (formación, intermediación, etc.) y por el otro, protegiesen al trabajador, en las situaciones de ausencia de trabajo asalariado, a través de prestaciones y subsidios de desempleo. Sin embargo, esta propuesta era sostenible por una concepción del desempleo como una cuestión friccional, un desajuste del mercado corregible desde visiones neoliberales. No obstante, es necesario destacar que otras perspectivas de corte marxista han planteado que el desempleo tiene un perfil estructural y cumple funciones consustanciales con el modo de organización capitalista. En cualquier caso, debemos señalar que las políticas de empleo no se concibieron como una herramienta de inserción desde la marginación, sino que se hizo des- de la situación de desempleo, permitiendo separar así a los excluidos de los desempleados (Varela y Álvarez-Uría, 1989; Santos, Montalbán y Moldes, 2004).

Este funcionamiento era sostenible por la política económica siempre que cuantitativamente las cifras de desempleo fuesen gobernables. Sin embargo, en el reciente contexto de intensificación del neoliberalismo, los datos de desempleo superan el 25 por ciento, que en números absolutos representan 5.904.700 desempleados en el Estado español (Encuesta de población activa del INE, 3t, 2013).

Cualitativamente el desempleo tiene un nuevo perfil en durabilidad que lo acerca a una condición estructural. El desempleo, para una buena parte de la población, ha dejado de ser una situación transitoria y se ha convertido en definitiva o de larga duración. Este hecho ha provocado que la distancia entre lo que históricamente se había concebido como marginal y aquello que se consideraba circunstancial se ha estrechado hasta tal punto que las fronteras de la exclusión social se extienden a un amplio número de población. Esta transformación se asemeja al paso de la pobreza marginal a la pobreza descalificadora, que señala Paugam (2007), donde:

Un mayor número de personas se enfrenta a situaciones de precariedad en el trabajo susceptibles de acumular varias desventajas: pocos ingresos, condiciones de vida y salud mediocres, fragilidad de la sociabilidad familiar y de las redes sociales de ayuda privada, participación incierta en cualquier tipo de vida social institucionalizada. [...] La pobreza descalificadora es una relación social con los «pobres» que genera una angustia colectiva porque cada vez más personas se consideran pertenecientes a esta categoría y muchas, cuya situación es inestable, temen que les suceda a ellas (p. 100).

En paralelo a esta realidad objetivable de carácter estructural, emergen algunas retóricas en las políticas de empleo basadas en la ficción del principio de igualdad de oportunidades, legitimadoras de la dominación y normalizadoras de la desigualdad. Estas posiciones ideológicas tienen sus efectos sobre la orientación de la política social hacia lógicas individualizadoras de los conflictos sociales y culpabilizadoras de las propias circunstancias a las personas que las padecen (Wacquant, 2010a y 2010b). 
Estas retóricas favorecen la desresponsabilización del Estado y las administraciones públicas de sus funciones garantistas de derechos, redistributivas y compensatorias. Tal y como señala Antonio Martínez López (2008), la fórmula para afrontar la moderna cuestión social pasa por un giro hacia un estado del bienestar activo, «donde la responsabilidad por la integración social tiende a descargarse progresivamente de las instancias públicas hacia el ámbito de los propios individuos y la sociedad civil» (p. 114). A través de metodologías de la «activación» o la «empleabilidad», por ejemplo, recuperadas de las actuales políticas de empleo, trasladan a cuestiones individuales-actitudinales las posibilidades de mejora de la propia situación, eximiendo la causalidad estructural, política y económica de cualquier responsabilidad con la sociedad y las personas (Serrano, Fernández y Artiaga, 2012).

\section{Método}

Este trabajo se organiza en dos etapas. La primera se nutre de la observación directa y la relación con profesionales, servicios y usuarios en el ámbito de la orientación para el empleo en Girona $^{1}$. En la segunda etapa se recurre a una revisión teórica de las políticas de empleo y a los cambios de orientación ideológica, a partir del análisis documental de normativas, recomendaciones y estrategias profesionales en Cataluña. Es por ello por lo que los datos de este trabajo son fundamentalmente cualitativos, los cuales nos permiten hacer un análisis de los discursos, de las prácticas profesionales, de las representaciones y de las experiencias del desempleo.

En este sentido, con este artículo se pretende problematizar la tendencia de cambio de orientación en las políticas sociales de empleo y sus posibles implicaciones en las prácticas profesionales. La experiencia profesional directa nos permite observar cómo las visiones individualizadoras depositan la responsabilidad de la inserción en criterios actitudinales que cada sujeto debe administrar.
¿Cuáles son los retos del Trabajo Social ante las necesidades de poblaciones no salarizadas cada vez más amplias? ¿Cómo atender a personas dependientes de subsidios no contributivos y compensatorios en Estados cuyas funciones garantistas cada vez son menores? ¿Cómo influyen los discursos en la construcción de soluciones individuales a cuestiones estructurales en las prácticas de intervención social? ¿Qué consecuencias tienen sobre los sujetos perceptores? ¿Cómo puede contribuir el Trabajo Social a reproducir o transformar estos discursos? Estas y otras preguntas son las que abordaremos en las siguientes páginas.

\section{Resultados y discusión}

\subsection{Nuevos paradigmas conceptuales}

Desde la Cumbre de Luxemburgo en 1997 y la Estrategia Europea para el Empleo de 2001 se introducen nuevos conceptos en los discursos del trabajo, la orientación y la inserción laboral que transforman las políticas de empleo de los Estados miembros ${ }^{2}$. Algunos de estos conceptos como: activación, empleabilidad, competencia, flexibilidad, y emprendiduría, no dejan de estar cargados de ideología y suponen una transformación en la mirada sobre las políticas de empleo, sobre las funciones de los organismos públicos, sobre los objetivos políticos y sobre distribución de las responsabilidades.

Esta nueva ideología es una aliada de las políticas sociales de corte neoliberal que acompañan al proceso de individualización y de retirada de las funciones compensatorias y redistributivas del Estado de bienestar. Esto da lugar a una nueva forma de protección social que pasa de la lógica del derecho a la lógica del merecimiento (Serrano, Fernández y Artiaga, 2012).

Retomando los conceptos, sujetos a discusión, que orientan las políticas de empleo, nos encontramos en primer lugar con el paradigma de la activación, que implica un nuevo replanteamiento entre los derechos y las responsabilida-

${ }^{1}$ La relación se estableció a partir de la actividad profesional de la autora principal de este artículo entre los años 2010 y 2011 en programas de Escuelas Taller y Casas de Oficio. Y entre 2013 y 2014 en un programa municipal de acompañamiento y orientación laboral a personas desempleadas. Ambas actividades se llevaron a cabo en dos municipios de la provincia de Girona.

2 Los cuatro pilares básicos de los que nacen las políticas de empleo de la Unión Europea son: Empleabilidad, desarrollo del espíritu de empresa, adaptabilidad e Igualdad de oportunidades (Santos, Montalbán y Moldes, 2004, pp. 187-188) 
des entre el desempleado y los poderes públicos. La noción de activación se presenta como una herramienta que pretende contrarrestar las políticas pasivas dedicadas a la transferencia de rentas a los desempleados y superar la lógica de la asistencia de los poderes públicos. De esta manera, se construye una ecuación en la que trabajador es igual a contribuyente y parado es igual a parásito, y contra esta idea se exige al desempleado la activación, que traslada al terreno de lo individual y lo actitudinal las posibilidades de incorporación al mercado de trabajo.

El nuevo paradigma de la activación, según los autores citados,

Apela a un cambio en el modo de gobierno, no solo del desempleo, sino también de las instituciones que intervienen para hacer frente a este problema. [...] El objetivo de la intervención serán las competencias, motivaciones y actitudes individuales, siendo el sujeto individual el eje nuclear de las intervenciones y por tanto parte del problema (pp. 44-45).

En esta línea, la cartera de servicios de Servei d'Ocupació de Catalunya (SOC), en lo referente a la orientación profesional, incluye el acuerdo y firma de un compromiso de actividad.

La articulación del servicio se basa en identificar la situación de la persona mediante el cuestionario de factores de ocupación. La identificación de ocupabilidad efectuada en base a este cuestionario supone la asignación de la persona a un grupo o colectivo con necesidades y potencialidades similares. La pertenencia a un grupo u otro delimita los servicios a los cuales deberá dirigirse esta persona para mejorarle la ocupabilidad y facilitarle la inserción en el mercado de trabajo, y permite establecer el Compromiso de Actividad de la persona usuaria de acuerdo con su colectivo (Pla de desenvolupament de les polítiques actives d'ocupació de Catalunya, 2012-2013)3.

Según el Glosario de las Políticas Activas de Ocupación de la Generalitat de Catalunya (2011, p. 52), el compromiso de actividad es el que «adquiere el solicitante o beneficiario de las prestaciones de buscar ocupación de una manera activa, aceptar una colocación adecuada y participar en acciones específicas de motivación, información, orientación, formación, reconversión o inserción profesional con la finalidad de incrementar su ocupabilidad». Su incumplimiento comporta efectos sancionadores como falta leve, a partir de los cien días de percepción de prestación.

Estos documentos técnicos, propios de los servicios competentes para el empleo, son portadores de otro concepto clave: la empleabilidad. La mejora de la empleabilidad es el objetivo básico de los servicios de ocupación. Se trata de un aspecto que incide en el cambio de funciones del Estado que ya «no puede asegurar el empleo pero debe asegurar la empleabilidad» (Santos, Montalbán y Moldes, 2004, p. 255). Es decir, el Estado debe garantizar que los trabajadores puedan ser empleables de acuerdo a un nuevo perfil de trabajador en el que priman cualidades personales por encima de las profesionales. El trabajador debe ser flexible, emprendedor y adaptable. En este sentido, nuevamente el «Glosario de las Políticas Activas de Ocupación de la Generalitat de Catalunya» define la ocupabilidad individual como:

Las posibilidades que posee una persona de encontrar trabajo y adecuarse al mercado de trabajo, teniendo en cuenta tanto sus aptitudes, es decir cualidades personales, como su currículum profesional. Por lo tanto, se centra, principalmente, en características intrínsecas de la persona, actitudes, aptitudes, que le facilitarán la consecución y el mantenimiento de un puesto de trabajo (2011, p. 125).

Para determinar la empleabilidad de los usuarios, los servicios de orientación, entre ellos el Servicio de Orientación Catalán, desarrollan un sistema clasificatorio de análisis de la empleabilidad de los usuarios a partir de factores estructurales, personales y competenciales (EMO/250/2012). De esta manera se generan diferentes tipologías de desempleados que permiten discernir entre empleables e inempleables. Esta actividad clasificatoria tiende a reproducir la selección del mercado de trabajo, así pues, son los propios servicios de empleo los que construyen a los inempleables al etiquetarlos y clasificarlos (Santos, Montalbán y Moldes, 2004, p. 254). El mo-

${ }^{3}$ Aprobado por el Consejo de Dirección del SOC en la sesión del 16 de febrero de 2012. Todos los documentos de la Generalitat de Cataluña expuestos en este artículo han sido traducidos en español por los autores del mismo. 
delo catalán, por ejemplo, define hasta un total de diez categorías de empleabilidad que se convierten en el punto de partida de los itinerarios de inserción laboral y que, a su vez, concentran el plan de mejora competencial.

La noción de competencia toma relevancia como el conjunto de capacidades, habilidades y destrezas que cada persona aprende a lo largo de su vida y puede aplicar en el ámbito laboral. El modelo por competencias es la metodología básica del Servicio de Orientación Catalán para el análisis de la ocupabilidad y establecimiento del itinerario de inserción laboral. Estas competencias se dividen en tres tipos según la clasificación del Istituto per lo Sviluppo della Formazione Professionale dei Lavoratori (ISFOL): de base, técnico-profesionales y transversales. Estas últimas, nuevamente, tienen que ver con aspectos actitudinales y de disposición para el empleo de los sujetos. Esta propuesta guarda relación con la idea de flexibilidad, que propone Richard Sennet, donde se rompen los vínculos y la identidad en el trabajo, y se produce una crisis del valor de la experiencia a favor de la adaptabilidad, la asunción del riesgo y la flexibilidad ante el trabajo. Estas cuestiones competenciales transversales despojan al trabajador del valor de la experiencia del trabajo y le exigen nuevas cuestiones actitudinales y morales, que lo asemejan a la idea de recurso humano.

Estos cambios, en las políticas de ocupación, hacia la activación, empleabilidad, flexibilidad, o la metodología por competencias ahondan en el compromiso del individuo ante lo que en realidad son conflictos estructurales. Se convierten así, en una forma de neobeneficencia que traslada de manera totalizadora la responsabilidad de su situación personal y las soluciones al desempleado. Prueba de ello son los eslóganes de algunos servicios municipales de ocupación como: «Tu puerta para la orientación, tú tienes la llave $\rangle^{4}$ del Espacio Municipal de ocupación del Ayuntamiento de Salt, Girona o «Para encontrar trabajo. Yo ya me he activado» del Ayuntamiento de Barcelona.

\subsection{Sujetos inempleables}

Una vez resueltos los índices de empleabilidad, cabe preguntarse quiénes son los sujetos inem- pleables. Robert Castel (p. 390) se ha referido a ellos como los supernumerarios, los trabajadores sin trabajo; Loïc Wacquant (2010b) habla del nuevo proletariado post-industrial que se encuentra fuera de las relaciones salariales; y Pierre Bourdieu los denomina el precariado.

Serge Ebersold (2001) genera la noción de inempleable para referirse precisamente a aquellas personas que carecen de los atributos propios de la empleabilidad, definidas por las políticas de ocupación atravesadas por las lógicas empresariales. Lo sujetos inempleables se construyen desde la perspectiva del déficit, ya sea de formación, de voluntad o de personalidad (Serrano et al., 2012, p. 45). Son personas carentes de las competencias propias que exige el mercado de trabajo. Este hecho contribuye a generar enfoques profesionales de corte patologizante y ponen en el centro de la intervención social la mejora de las disfunciones individuales del desempleado. Los inempleables no solo atentan contra las lógicas del mercado, sino que también lo hacen contra la moralidad hegemónica, ya que las normas del mercado de trabajo se han convertido en normas sociales compartidas por el conjunto de la población, en el punto de partida para la aceptación social. De esta manera, se reconstruye al sujeto perceptor como presunto fraudulento, abusador y «aprovechado», alejando el centro de la cuestión de la desigual distribución de riqueza y la acumulación privada, hacia la perniciosa culpabilización del «malgasto» público. En este sentido Serrano, Fernández y Artiaga señalan que el nuevo Estado social tiene que combatir la dependencia como situación, y la pasividad, como actitud (p. 45). Un aspecto que Sennet presenta a partir de su propuesta de la ideología del parasitismo social y del desprestigio de la dependencia.

En los procesos de selección de usuarios se utiliza frecuentemente la noción de persona insertable, que responde a la idea del buen parado (Santos, Montalbán y Moldes, 2004, p. 274). Es decir, para referirse a aquellas personas que tienen una respuesta positiva ante la formación, motivadas ante cualquier recurso, con hábitos laborales y sociales, etc.; que son una garantía para la consecución de los objetivos de los programas de orientación. Sin embargo, los inserta-

${ }^{4}$ [La teva porta per a l'orientació. Tu n'ets la clau]. En catalán la palabra clau tiene dos significados: llave y clave, por lo tanto se le asigna a la persona desempleada su doble significación. 
bles no dejan de ser figuras sometidas a las dinámicas del mercado. Quienes hace unos años podían cambiar de trabajo sin grandes dificultades en el ámbito de la construcción, por ejemplo, hoy se convierten en personas inempleables. Al mismo tiempo, las personas con un alto nivel de formación que aparentemente cuentan con un elevado nivel de empleabilidad, también tienen severas dificultades para la inserción laboral y no encuentran en los servicios públicos de ocupación programas que respondan a sus intereses y necesidades, ya que no cumplen la lógica del déficit.

Esta carga conceptual que rodea las políticas de empleo convierte a la persona desempleada en responsable de su fracaso, de su situación. Recupera los discursos utilizados en otras épocas en el ámbito de la pobreza y las traslada al ámbito de las políticas de empleo. Deja en manos del trabajador la responsabilidad de encontrar un trabajo, pero ignora la realidad socioeconómica que condiciona su situación. Así, la responsabilidad estatal ya no pasa por establecer políticas de empleo que favorezcan una integración laboral con garantías de calidad, seguridad o estabilidad, sino simplemente por favorecer los cambios legislativos que hagan más atractiva la contratación para los empresarios, puesto que los aspectos cualitativos de lo que significa tener un trabajo ya no le incumben al Estado.

\subsection{La práctica profesional ante el para- digma de la activación y la empleabilidad}

Vemos que todos estos acontecimientos abren un nuevo escenario de reflexión ético-profesional en torno a las orientaciones y las prácticas de las políticas sociales. El Trabajo Social juega un papel fundamental en sus posibilidades de reproducción o transformación de la realidad social. Las prácticas profesionales se ven notablemente limitadas por las políticas de recortes en prestaciones, personal, servicios e infraestructura que obedecen a un cambio de modelo del Estado de bienestar (Adelantado, s.f.). Se trata de un cambio que conduce a una remercantilización de lo que, en modelos anteriores, se consideraban derechos sociales. Es decir, es el ámbito de los derechos sociales y del bienestar donde el capital busca un nuevo espacio de mercado. Ahora bien, para llevar a cabo este cambio es necesario acompañarlo con nuevos discursos que calen en la sociedad y en la acción profesio- nal. En este sentido los profesionales de la acción social jugarán un rol fundamental que pasa inevitablemente por reproducir los discursos individualizadores. De este modo, la práctica profesional no se ve únicamente limitada por los recortes o aspectos cuantitativos, sino también abrumada por aspectos cualitativos como son los nuevos discursos que pueden transmitir o reproducir.

El caso de la empleabilidad como objetivo de intervención o de acciones, como la mejora de competencias personales hacia el trabajo, recalan en la consideración individual del conflicto que genera el paro. Los diferentes programas previstos desde las políticas activas de ocupación y los ejercicios de clasificación, como hemos mencionado anteriormente, suelen responder a las capacidades de los servicios y las instituciones más que a las necesidades de las personas a las que atienden. El diseño de los programas se hace a la medida de las posibilidades que pueden asumir el contexto territorial y los servicios; así la mayoría de programas se ocuparán de aquellas personas desempleadas consideradas insertables, según criterio institucional a demanda del mercado, de manera que los profesionales de la inserción social se acaban convirtiendo en pseudodispositivos de selección de personal para el sector empresarial y al mismo tiempo generan vacíos en relación a determinados grupos sociales que quedan sin atender.

Por otro lado, cuando el único objetivo es la vuelta al empleo, no se cuestionan las condiciones del trabajo. De alguna manera las nuevas pautas de intervención social son también un mecanismo que permite educar en la precariedad y convierten en aceptables las situaciones de explotación laboral: eventualidad, flexibilidad, restricciones contractuales, bajos salarios, infra-ocupación, etc.

\section{Conclusiones}

En el modelo anterior, la inserción laboral era en un objetivo fundamental de la intervención social $\mathrm{y}$, en buena medida, en la contrapartida de las políticas de bienestar. Esta estrategia daba fe de que el trabajo era más que trabajo y el no trabajo era más que desempleo, como sostiene Castel. Así el trabajo asalariado apareció como un elemento central de la vida y de la participación y relación social, la vía de acceso al consumo de masas de 
productos estandarizados y organizados por razón de clase (Beck, 2009). Al mismo tiempo se convierte en generador de hábitos, pautas de relación, conductas y comportamientos que devienen, al fin y al cabo, mecanismos de disciplinarización social (Foucault, 1979). Nos encontramos ante un escenario en el que el trabajo ha dejado de desempeñar esta función, precisamente porque los puestos de trabajo se han reducido notablemente y buena parte de los existentes han sufrido una precariedad generalizada.

En paralelo, el Estado de bienestar ha disminuido, en buena medida, las funciones redistributivas que se le encomendaron, y las exigencias del capitalismo lo están transformando en un nuevo paradigma. Este cambio se apoya en las dos estrategias, una discursiva y otra de intervención social, que las hemos ido caracterizando a lo largo de este artículo.

Estos cambios ponen en el centro del debate las condiciones materiales de la existencia, cuando no las garantiza ni el trabajo asalariado ni la acción redistributiva del Estado. Esta situación plantea nuevos retos al Trabajo Social y a otras disciplinas de la intervención social, que tienen que ver con dos elementos claves de la profesión: por un lado, los aspectos metodológicos, y por el otro, los políticos. En lo que respecta a los aspectos metodológicos los profesionales de la intervención social deberíamos revisar críticamente algunas de nuestras prácticas que tienen que ver con:

a) Analizar las causas de tipo estructural de las problemáticas y no actuar únicamente sobre los efectos. Una lectura causal permite generar conciencia de los factores y dinámicas estructurales que producen determinadas situaciones de desigualdad y vulnerabilidad. Esta conciencia debe compartirse con la sociedad para poder incidir en ella de manera transformadora.

b) Distanciarse de actitudes de culpabilización. Es decir, la práctica profesional no puede explicar el desempleo únicamente a partir de cuestiones biográficas o actitudinales. En este sentido, cuando el principio de redistribución se ve limitado, las soluciones al problema no deben pasar por el manejo de cuestiones motivacionales. Este tipo de actuaciones profesionales refuerzan la culpabilización y la patologización.

c) Desvincularse de las prácticas de control social. Evitar caer en la lógica de la activación que presupone la pasividad de la persona desempleada. Esta lógica que propone constantes acciones formativas, mejoras de competencias, cursos y «cursillitos», no responde, en algunas ocasiones, a los intereses del usuario ni del mercado del trabajo y se convierten únicamente en un mecanismo de control que permite evaluar el desarrollo del programa pero no su impacto en la vida de las personas.

d) Visión holística de las problemáticas. Capacidad de situar al individuo en su entorno. Es decir, se trata de superar la confusión entre acción individual e individualizadora. No hay duda de que los profesionales del Trabajo Social debemos ser capaces de abordar las problemáticas desde la perspectiva de casos; sin embargo es un error hacer de la situación particular una lectura total.

e) Reforzar la concepción de los usuarios como sujetos de derecho en un contexto en el que los derechos sociales están seriamente amenazados. Los trabajadores sociales no pueden ser portadores del discurso del recorte ni justificar las actuaciones basadas en el merecimiento.

En lo político, cabría recuperar los debates y las propuestas que reivindican nuevas formas de redistribución de la riqueza que permitan garantizar las condiciones materiales de la existencia como un derecho universal, donde el trabajo asalariado pasa de ser una obligación a una elección. Ya que la supervivencia material es una condición básica para la justicia social y la libertad. En este sentido, el Trabajo Social no debe renunciar a ser una ciencia social posicionada.

\section{Referencias bibliográficas}

Adelantado, J. (s.f.). L'estat de benestar: retallades i ¿canvi de paradigma? El cas espanyol (19952012). En A. Tarabini, R. Borras y A. Grimalt (eds.), Cap a on anam? Els ciutadans de les Illes Balears. Palma de Mallorca: Fundació Gadeso.

Beck, U. (2009). La sociedad del riesgo. Hacia una nueva modernidad. Barcelona: Paidós.

Bourdieu, P. (1999). Contrafuegos. Reflexiones para servir a la resistencia contra la invasión neoliberal. Barcelona: Anagrama

Castel, R. (1997). Las metamorfosis de la cuestión social. Una crónica del salariado. Buenos Aires: Paidós 
BOGC. (2012). ORDRE EMO/250/2012, de 28 d'agost, per la qual s'estableixen les bases reguladores i sobre la convocatòria per a l'any 2012 per a la concessió de subvencions per a la realització d'accions d'orientació i acompanyament a la inserció de la xarxa d'orientació per a l'ocupació. BOGC, 6207, 41922-41949.

Ebersold, S. (2001). La naissance de l'inemployable. Rennes: Presses Universitaires de Rennes.

Foucault, M. (1979). Vigilar y castigar. Madrid: Siglo XXI

Martínez López, A. (2008). La influencia de las políticas activas de empleo en las transformaciones de los modos de intervención y los modelos contemporáneos de bienestar social. Portularia. Revista de Trabajo Social, VIII(2), 103-115.

Paugam, S. (2007). Las formas elementales de la pobreza. Madrid: Alianza

Santos, A., Montalbán, C. y Moldes, R. (2004). Paro, exclusión y políticas de empleo. Aspectos sociológicos. Valencia: Tirant lo Blanch

Sennet, R. (2000). La corrosión del carácter: las consecuencias personales del trabajo en el nuevo capitalismo. Barcelona: Anagrama.

Serrano, A., Fernández, C. J. y Artiaga, A. (2012). Ingenierías de la subjetividad: el caso de la orientación para el empleo. Reis. Revista Española de Investigaciones Sociológicas, 138, 41-62. doi: $10.5477 /$ cis/reis. 138.41 .

Servei d'Ocupació de Catalunya. (2011). Glossari de Politiques Actives d'Ocupació. Tercera versión. Recuperado de: http://www.oficinadetreball.cat/socweb/export/sites/default/socweb_ca/auxiliar/_fitxers/PolitiquesActivesOcupacio_Glossari.pdf (Consiltado el 29 de diciembre de 2013)

Servei d'Ocupació de Catalunya. (2012). Pla de Desenvolupament de les políticas Actives d'ocupació de Catalunya (PDPA) 2012-2013. Aprobado por el Consejo de Dirección del SOC en la sesión del 16 de febrero de 2012. http://www.oficinadetreball.cat/socweb/export/sites/default/socweb_ca/web_institucional/_fitxers/PDPA_CDSOC_16_02_definitiu.pdf (Consultado el 29 de diciembre de 2013)

Varela, J. y Álvarez-Uría, F. (1989). Sujetos frágiles: ensayos de sociología de la desviación. Mexico: Fondo de Cultura Económica.

Wacquant, L. (2010a). Castigar a los pobres. El gobierno neoliberal de la inseguridad social. Barcelona: Gedisa.

Wacquant, L. (2010b). Parias urbanos. Marginalidad en la ciudad a comienzos del milenio. Buenos Aires: Manantial 